\title{
ACANTHOBOTHROIDES PERUENSIS N. SP. (TETRAPHYLLIDEA: ONCHOBOTHRIIDAE) PARÁSITO DE DASYATIS BREVIS G. (RAJIFORMES: DASYATIDAE) DE LA COSTA DEL PERÚ.
}

ESPERANZA LOPEZ C.

\author{
Facultad de Ciencias Biológicas. Universidad Ricardo Palma. Apart. 138. Lima - Perú
}

\begin{abstract}
RESUMEN
Se describe una nueva especie. Acanthobothroides peruensis n. sp., párasito de Dasyatis brevis (Dasyatidae) pez elasmobranquio de la costa peruana. Caracterizada por el número de testículos (120-140) y su distribución en todo el proglótido y por el tamaño y relación entre los ganchos mediales y laterales del escolex (2.7:1)

Palabras Claves: Acanthobothroides peruensis n. sp., Dasyatis brevis, Costa peruana.
\end{abstract}

\section{INTRODUCCION}

En el Perú se conoce poco acerca de la fauna parasitaria de peces marinos, sobre todo en cestodes. Al revisar tractos digestivos de Dasyatis brevis, encontramos céstodes correspondientes al género Acanthobothroides cuyas características son diferentes de A. thorsoni. Registramos este género para el Pacífico y se describe A. peruensis n. sp.

\section{MATERIAL Y METODOS}

Los huéspedes se obtuvieron durante los meses de Enero a Marzo de 1988; cuatro procedían de Chimbote (90 05'.Lat. Sur y 78 $33^{\prime}$ Long. Oeste). Para la identificación de estos huéspedes se utilizó la clave de Chirichigno (1974).

Los parásitos fueron separados vivos de la válvula espiral del huésped para ser colocados en agua de mar y fijados en formalina al $10 \%$, previo aplastamiento entre dos láminas porta objetos, cloreados con carmín acético de Semichon o hematoxilina de Delafield, deshidratados en pasos sucesivos hasta alcohol absoluto, aclarados en xilol y montados en Bálsamo de Canadá; los dibujos se hicieron utilizando una cámara lúcida Leitz y las medidas con un ocular micrométrico calibrado, expresándose en mílimetros salvo que se indique otra forma; primero se anota el promedio y luego el ámbito entre paréntesis. Para la descripción de los ganchos se empleó la terminología de Caira (1985)
Las siglas MHJP e IMT corresponden a las colecciones Helmintológicas del Museo de Historia Natural «Javier Prado» y del Instituto de Medicina «Daniel A. Carrión», ambas pertenecientes a la Universidad Nacional Mayor de San Marcos, Lima Perú.

\section{RESULTADOS}

De los 10 especímenes de D. brevis, $8(80 \%)$ estuvieron parasitados con A.peruensis n. sp. El número de especímenes por cada pez fué de 3 a 15 .

FAMILIA: ONCHOBOTHRIIDAE Braun, 1900 Acanthobothroides peruensis n. sp. (Figs. 1-4)

DESCRIPCION: Basada en 14 especímenes coloreados. $14 \mathrm{~cm}$. de longitud; 4 botridios, triloculados con ventosa anterior y un par de ganchos, siendo el medial grande y simple, el lateral pequeño y bífido; ganchos mediales de base ancha y punta curva en ángulo recto, $0.19(0.18-0.20)$ de longuitud, los ganchos laterales se midieron con dificultad porque sus prolongaciones axiales y abaxiales se encontraron incompletas, en los escólices fijados «in situ» miden $0.06(0.060-0.070)$.

Lóculo anterior del botridio de $0.56(0.54-0.59)$ de longitud; el lóculo medio de $0.50(0.48-0.52)$ y el posterior de $0.50(0.45-0.54)$

Escolex 1.41 (1.35-1.42) de longitud por 1.77 (1.72-1.80) de ancho. Pedúnculo cefálico robusto, 4 

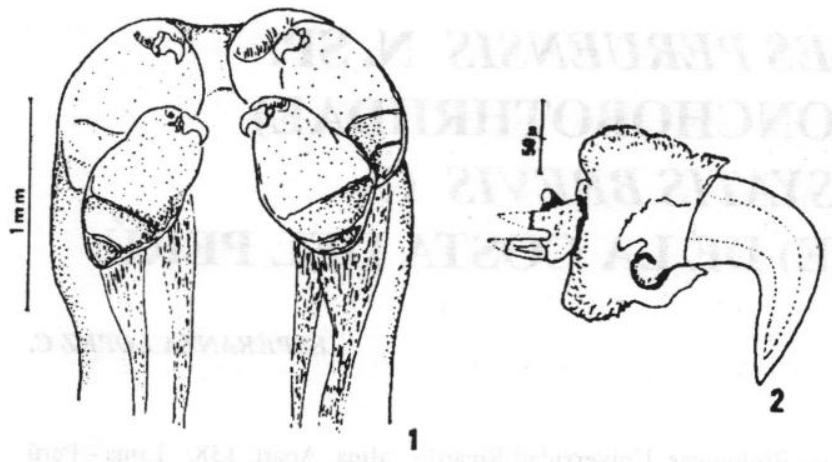

Fig. 1. Escolex de A. peruensis.

Fig. 2. Gancho medial y lateral de A. peruensis.

$(3.8-4.2)$ con 4 haces musculares muy desarrollados.

Proglótidos maduros más anchos que largos o cuadrados, $0.80(0.78-1.10)$ de largo, $2.95(2.90-3.05)$ de ancho. Proglótidos grávidos más anchos que largos 1.25 (1.12-1.27) de longitud, 2.83 (2.82-2.84) de ancho.

Poros genitales irregularmente alternos, se abren aproximadamente en la mitad superior y lateral del segmento. Testículos casi esféricos en número de 135 (120-140), distribuidos en todo el proglótido, 0.10 (0.090.12 ) de diámetro.

Saco del cirro ovoide, ligeramente arqueado hacia la región anterior, $0.19(0.18-0.20)$ de longitud por 0.12 (0.11-0.13) de ancho; cirro espinoso. Vesícula seminal externa bien desarrollada, formando numerosas circunvoluciones. Ovario bilobulado, se sitúa en la parte media posterior del proglótido. Vagina anterior al saco del cirro; glándulas vitelógenas foliculares y laterales. Utero sacciforme y con lobulaciones laterales.

Sitio de Infección: Válvula espiral.

Huésped: Dasyatis brevis $\mathrm{G}$.

Holotipo: Col. Helm. MHJP No. 1016.

Paratipo: Col. Helm. IMT No. 674.

\section{DISCUSION}

Brooks (1977) propuso el género Acanthobothroides con la especie A. thorsoni, parásito de Himantura schamardae (Werner, 1904), pez elasmobranquio de la familia Dasyatidae capturado en el mar del Caribe, Colombia.

Posteriormente, Tantaleán y Rodríguez (1987) refierieron su hallazgo en Dasyatis brevis de la costa de Chimbote (Ancash) indicando que presentan diferencias con la descripción original, las cuales atribuyen a variaciones intraespecíficas.

Sin embargo, al estudiar nuevo material llegamos a la conclusión de que se trata de una nueva especie para lo cual tomamos en cuenta los criterios de Ostrowsky de Nuñez (1973) quien manifiesta que el aumento de los órganos sexuales es el camino a la especiación. $A$. peruensis n. sp. se diferencia de A. thorsoni por lo siguiente:

1) Número de los testículos, que en $A$. peruensis es mayor (120-140) que en A. thorsoni (87-97).

2) En la distribución de los testículos, que en la nueva especie abarca todo el segmento, incluyendo la zona de los lóbulos del ovario; en cambio, en A. thorsoni los testículos ocupan sólo la mitad anterior.

3) Tamaño de los ganchos mediales y laterales. En A. peruensis n. sp. son más pequeños, miden 0.19 y 0.06 (2.7: 1 ); en cambio, en A.thorsoni miden $0.22-0.28$ y 0.16 $0.19(1.4: 1)$

Por estas consideraciones, creemos que la especie estudiada es nueva y la nominamos como Acanthobothroides peruensis $\mathrm{n}$. $\mathrm{sp}$.
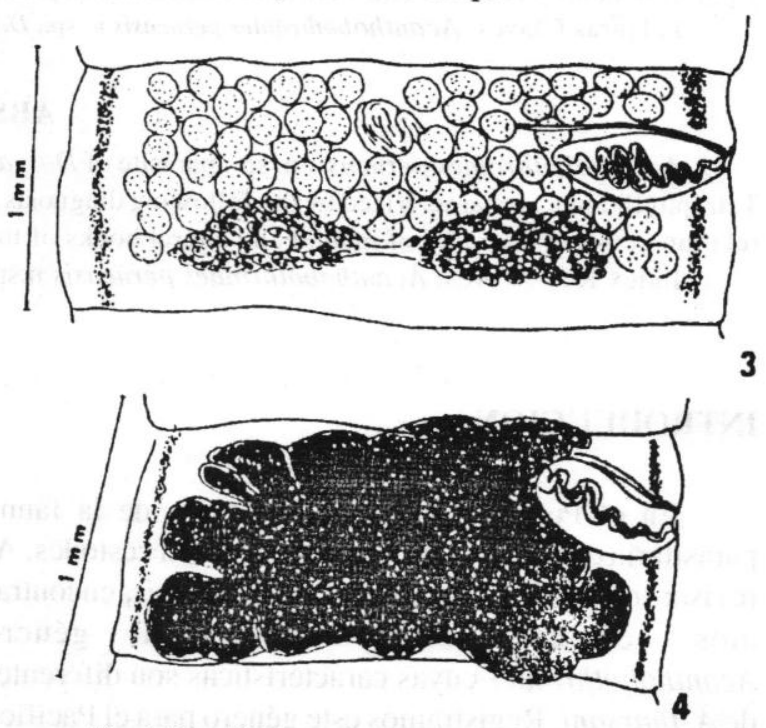

Fig. 3. Proglótido maduro de A. peruensis. Fig. 4. Proglótido gravido de $A$. peruensis.

\section{REFERENCIAS BIBLIOGRAFICAS}

BROOKS, D.R. 1977 Six new species of Tetraphyllidean Cestodes including a new genus, from a marine Stingray Himantura schamardae (Werner 1904) from Colombia. Proc. Helminthol. Soc. Wash. 44: 51-59

CAIRA, J.N. 1985 Calliobothrium evani n. sp. (Tetraphyllidea: Onchobothriidae) from de Gulf of California with a redescription of the hooks of C. lintoni. Proposal for Onchobotriid hook termilogy. Proc. Helmintol. Soc. Wash. 52: $166-174$

OSTROWOSKY DE NUÑEZ, M. 1973. Estudios preliminares sobre la fauna parasitaria de algunos elasmobranquios del litoral Bonaerense, Mar del Plata, Argentina. 11. Cestodes de Mustelus schmitti Springer, 1939. Physis, 32: $1-14$.

TANTALEÁN, V. M. Y RODRÍGUEZ, J. 1987. Nuevos registros de helmintos parásitos de peces elasmobranquios de la costa del Perú. Rev. Biol. Trop. 35: 167-168.

SOUTHWELL, T. 1925. A monograph on the Tetraphyllidea with notes on related cestodes. Mem. Liverpool School. Trop. Med. New Series, 2. 368 pp. 